University of Nebraska - Lincoln

DigitalCommons@University of Nebraska - Lincoln

$11-1-2004$

\title{
A Comparison of Performance on the Towers of London and Hanoi in Young Children
}

\author{
Rebecca Bull \\ University of Aberdeen, United Kingdom, r.bull@abdn.ac.uk \\ Kimberly A. Espy \\ University of Nebraska-Lincoln, kespy2@unl.edu \\ Theresa E. Senn \\ Southern Illinois University School of Medicine
}

Follow this and additional works at: https://digitalcommons.unl.edu/dcnlfacpub

Part of the Neurosciences Commons

Bull, Rebecca; Espy, Kimberly A.; and Senn, Theresa E., "A Comparison of Performance on the Towers of London and Hanoi in Young Children" (2004). Developmental Cognitive Neuroscience Laboratory - Faculty and Staff Publications. 11.

https://digitalcommons.unl.edu/dcnlfacpub/11

This Article is brought to you for free and open access by the Developmental Cognitive Neuroscience Laboratory at DigitalCommons@University of Nebraska - Lincoln. It has been accepted for inclusion in Developmental Cognitive Neuroscience Laboratory - Faculty and Staff Publications by an authorized administrator of DigitalCommons@University of Nebraska - Lincoln. 


\title{
A Comparison of Performance on the Towers of London and Hanoi in Young Children
}

\author{
Rebecca Bull, ${ }^{1}$ Kimberly Andrews Espy, ${ }^{2}$ and Theresa E. Senn ${ }^{2}$ \\ ${ }^{1}$ Department of Psychology, University of Aberdeen, Scotland \\ ${ }^{2}$ Department of Family \& Community Medicine, Southern Illinois University School of Medicine, USA
}

\begin{abstract}
Background: The Towers of London (TOL) and Hanoi (TOH) have been viewed as equivalent measures of planning and/or problem solving, although recent evidence in adults suggests that the underlying measurement characteristics of these two tasks may differ. As tower tasks are one of the few instruments that can be used to assess executive functioning in young children, the cognitive demands for both tasks merit further examination. Methods: The relation among tower tasks and those of short-term memory, inhibition, and shifting ability were examined in a sample of 118 typically developing young children ( $M$ age $=4$ years, 9 months, $S D=6$ months). Half the children completed TOL and half completed TOH, with groups matched with respect to age, sex, and child vocabulary. Results: Whilst performance on a shifting task uniquely predicted TOH performance, none of the executive function measures were related to TOL performance after statistically controlling for the influence of baseline naming speed. For both tower tasks, performance on a shifting task contributed more strongly on complex trials that required more moves in the counter-intuitive direction relative to the end-state goal, whereas inhibition task performance only predicted performance on complex TOL trials. Conclusions: Successful tower task performance may be determined, at least at higher levels of complexity, by mental flexibility in this age range. However, overall the findings suggest that TOL and TOH are not interchangeable tasks even in young children, and more generally, raise methodological issues regarding the complex nature of executive function tasks.
\end{abstract}

Keywords: Executive functions, inhibition, shifting, short-term memory, tower tasks, children.

Abbreviations: TOL: Tower of London; TOH: Tower of Hanoi; EF: executive functions.

Although there remains considerable debate as to precisely what cognitive abilities comprise executive functioning (EF; Lyon \& Krasnegor, 1996), commonly identified component processes include: the ability to inhibit irrelevant information or task sets, shifting set or mental flexibility, and the ability to hold and update information in working memory (Baddeley, 1996; Miyake et al., 2000). Generally, EF encompasses the skills necessary for purposeful, goal-directed activity across time or task demands (Lezak, 1993; Shallice, 1990; Stuss, 1992). EF plays an important role in many aspects of child development, particularly with regard to certain developmental disorders such as autism and attention deficit hyperactivity disorder (Ozonoff \& Jensen, 1999), prematurity (Espy et al., 2003), and in relation to learning difficulties associated with reading, comprehension, and mathematics (Bull, Johnston, \& Roy, 1999; Bull \& Scerif, 2001; Gathercole \& Pickering, 2000; Swanson \& Ashbaker, 2000; Swanson, Ashbaker, \& Lee, 1996). Furthermore, different disorders likely yield systematic differences in the pattern of strengths and weaknesses in EF (Pennington, 1997).

EF has been linked extensively to the prefrontal cortex and related subcortical systems. Prefrontal systems are relatively immature during childhood and show continued, protracted development into early adolescence (Huttenlocher \& Dabholkar, 1997; Kinney, Brody, Kloman, \& Gilles, 1988; Thatcher, 1991), providing sup- port for the protracted development of executive abilities (Welsh, Pennington, \& Grossier, 1991). However, the relation between brain structure/function and EF test performance is not isomorphic in adults, and has not been investigated thoroughly in children. For example, performance on the Wisconsin Card Sorting Test (Heaton, Chelune, Talley, Kay, \& Curtis, 1993), often considered the 'classic' measure of EF and correspondingly of the prefrontal lobes, did not differ between children with defined lesions to the prefrontal cortex and those with lesions to other brain areas (Chase-Carmichael, Ris, Weber, \& Schefft, 1999). In contrast, frontal lobe lesion volume is related to performance on spatial mazes (Levin, Song, Ewing-Cobbs, \& Roberson, 2001) and word fluency tasks (Levin, Song, Ewing Cobbs, Chapman, \& Mendelsohn, 2001) in children who incurred traumatic brain injuries. What can be missed in the interpretation of these mixed findings is that the observation of any putative group differences on a measure of interest is affected by the measurement characteristics of the EF test. In most modern theories, EF is viewed as fractionated, composed of several interrelated, multi-dimensional 'executive' processes that recruit and exert higher-order control over more modular abilities, such as language or visuo-spatial skills. Therefore, tasks that measure welldefined cognitive processes will yield more homogeneous patterns of outcome following injury. Before es- 
tablishing a link to brain structure or function, a more thorough understanding of the specific cognitive components that comprise EF task performance is critical in order to make valid brain-behavior inferences.

This problem is even more salient when considering brain-behavior relations in young children. Many scientists consider cognitive abilities to be less differentiated in younger children; therefore, the nature of the relations between cognitive components and task performance is more complicated. The study of EF has been largely limited to children 6 years and older due to the lack of standardized measures that are applicable for use with younger children (Espy, 1997; Espy, Kaufmann, McDiarmid, \& Glisky, 2001). Yet, many of the disorders that affect outcome in school age children actually become manifest during the preschool period (e.g., attention deficit hyperactivity disorder). Furthermore, young children who incur brain damage or exhibit brain dysfunction have executive skill deficits that may attenuate their early cognitive development and subsequent academic proficiency (Anderson, 1998; Espy et al., in press; Welsh et al., 1991). Therefore, developing new assessment tools for use in younger children and understanding the contributors to performance is imperative in order to be able to identify putative EF deficits earlier in development.

The purpose of this paper is to assess the cognitive demands of two executive function tasks used extensively with adults, which also can be used with preschool and young children, namely the Tower of London (TOL; Shallice, 1982) and Tower of Hanoi (TOH; Simon, 1975). Although performance on both tasks is related to prefrontal function in adults, demonstrated both by frontal lobe lesion studies (Carlin et al., 2000; Owen, Downes, Sahakian, Polkey, \& Robbins, 1990) and investigations using imaging procedures to measure activation during problem solution in normals (Baker et al., 1996; Morris, Ahmed, Syed, \& Toone, 1993; Rowe, Owen, Johnsrude, \& Passingham, 2001; van den Heuvel et al., 2003), it is not clear whether both tasks measure the same executive processes. Clinicians and researchers treat the tasks as interchangeable or isomorphic, yet this view has not been validated empirically, particularly in children. One underutilized method to investigate such psychometric issues is to administer each pertinent task to a unique sample, but also to administer common criterion validity measures of interest to both samples. This method eliminates any interference among the pertinent tasks of interest had they been administered to the same sample, yet allows comparison of relative relations of task performance with other criterion measures. In light of the multifaceted nature of executive functions, the demand characteristics of the tower tasks merit further investigation.

On first inspection, TOL and TOH share many surface similarities. They both require the transfer of objects (balls and disks, respectively) on pegs from an initial start state into a goal end-state in the minimum number of moves. Furthermore, both tasks impose some common rules restricting the manner in which the objects can be moved from peg to peg. Only one ball/disk can be moved at a time, and any ball/disk not being moved must remain on a peg (see Figure 1 for examples of both TOL and TOH configurations). Based on these task similarities, successful tower performance would appear to require formulating a planned sequence of moves, retaining the plan, executing the moves, and monitoring and revising of the plan in advance of, or subsequent to, action.

Several cognitive processes likely contribute to tower performance, working memory being the most obvious (Phillips, Wynn, Gilhooly, Della Sala, \& Logie, 1999). In addition, Klahr (1994) has noted the important role of counter-intuitive moves in tower performance. Counter-intuitive moves are those that are in the direction away from the end-state goal, requiring planning and inhibition of the 'prepotent' move that is in the direction of the end-state goal. These 'prepotent' moves are problematic as the participant must make excess moves to reproduce the desired end-state configuration, and this ultimately may lead to an incorrect solution of the individual tower problem. Mental flexibility or shifting also may be important, as the participant must shift flexibly among subgoals or moves to achieve the configuration. As such, TOL and TOH generally are described as higher-order planning tasks because successful completion requires the participant to 'look ahead' and solve the problem cognitively before actually moving the balls or disks.

However, tower tasks need not be solved by 'look ahead' planning; but rather, can be completed using a more real-time, 'perceptual' strategy. Such participants use more direct, on-line processing, where the current object configuration immediately guides the next move. That is, the participant tries to bring the configuration successively closer to the end-goal state with each move, rather than a period of pre-planning followed by plan implementation. Therefore, inhibitory processes may be particularly important when such on-line perceptual strategies are used for tower problem solution (Goel \& Grafman, 1995; Goel, Pullara, \& Grafman, 2001; Miyake et al., 2000).

Despite the superficial similarities in structural features and cognitive demands for successful tower performance, the overlap between tower task performance in adults is not as close as one might imagine. Minor differences in structural features may contribute to different task demands. In TOL, the size of the pegs constrains the number of balls that can fit on each peg, whereas in $\mathrm{TOH}$ the diameter of the peg constrains the order of disk placement. The administration directions also differ between the two tower tasks. In $\mathrm{TOH}$, participants are not told the minimum number of moves required for each trial; 

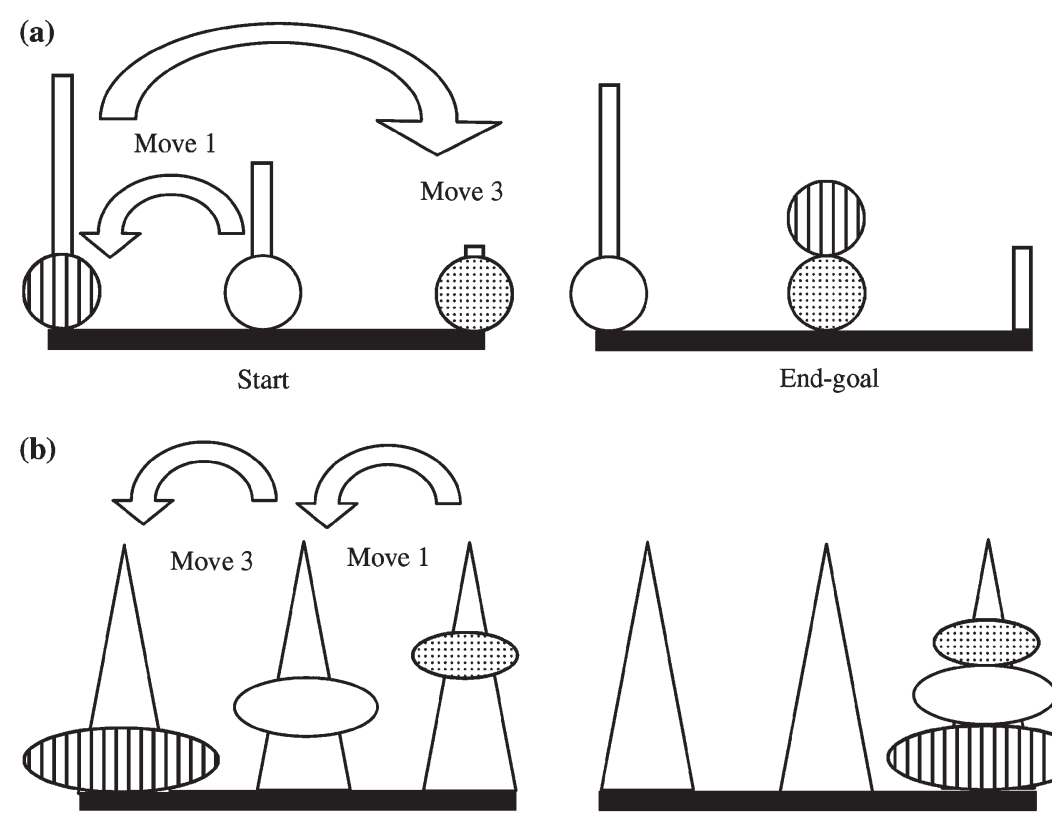

Start

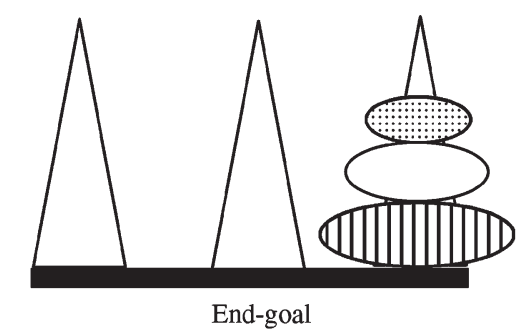

Figure 1. Examples of TOL (a) and TOH (b) trials showing initial object positions and the end goal. Both examples show 5-move problems requiring the participant to make two counter-intuitive moves ('goal-subgoal conflicts'). The arrows show the two counter-intuitive moves (i.e., moves that are in opposition to the end-state goal) that must be made in each Tower task

whereas in TOL, participants are instructed to achieve the configuration in a specified minimum number of moves for each trial, as quickly as possible. This instructional difference may influence more strongly the participant to begin moving the balls in TOL before any planning is undertaken, and may promote less on-line monitoring of the number of moves made in TOH. Finally, a physical model of the end-state goal as presented in $\mathrm{TOH}$, but not TOL where the end-state goal is represented pictorially, may make the goal more salient, particularly to younger children. Quite clearly, whilst both of these tasks are used as measures of planning ability, these differences may result in the use of different strategies for solution between the two tower tasks, particularly with respect to planning, and may engender unique cognitive demands for each task (see also Goel \& Grafman, 1995). Therefore, success or failure on each task may not be resultant upon the same underlying cognitive skills.

Welsh and colleagues reported correlations between TOL and TOH performance ranging from .37 (Humes, Welsh, Retzlaff, \& Cookson, 1997) to .61 (Welsh, Satterlee-Cartmel, \& Stine, 1999), with up to $86 \%$ of the variance not shared between the tower tasks. Using the task comparison method described above in adults, Welsh et al. (1999) found that whilst working memory and inhibition strongly predicted TOL performance, inhibition and perhaps a processing speed factor were related only weakly to $\mathrm{TOH}$ performance in adults. Little $\mathrm{TOH}$ performance variability was accounted for by working memory. In school age children and adolescents, Bishop, Aamodt-Leaper, Creswell, McGurk, and Skuse
(2001) found that inhibition was unrelated to TOH performance. Bishop et al. suggested that shifting between different subgoals (i.e., a conscious inhibition of a previous subgoal with flexible switching to an alternative subgoal) may be a better predictor of $\mathrm{TOH}$ performance, rather than inhibition of automatically activated prepotent information, measured by various Stroop-like tasks.

Understanding these issues in young children, in whom executive functions and prefrontal systems undergo significant maturation in this developmental period, will allow a more thorough, ontogenetic view, when considered in light of findings from older school age children (e.g., Bishop et al., 2001) and adult (e.g., Welsh et al., 1999; Humes et al., 1997) samples. The use of more perceptual on-line strategies to solve the tower problems, which may rely more on inhibition, likely are particularly prominent in young children who have more limited cognitive capacity and/or metacognitive skills (Flavell \& Wellman, 1977). Young children typically do not demonstrate overt behaviors that are considered 'planful', as they do not pause before moving the disks/balls or do not appear to be implementing systematic move sequences. Because inhibition may be particularly important when tower tasks are being solved using such on-line perceptual strategies (Goel \& Grafman, 1995; Goel et al., 2001; Miyake et al., 2000), inhibitory processes may be a stronger contributor to $\mathrm{TOH}$ and TOL performance in younger children, in comparison to that of older children and adults. In particular, the relative contributions of inhibition, shifting, and short-term memory to performance on each tower task 
was examined in young children, including analysis of contributions to performance on the complex problems that vary in the number of counter-intuitive moves.

\section{Method}

\section{Participants}

One-hundred and eighteen children participated in the study, ranging in age from 3 years 7 months to 6 years 9 months $(M=4$ years, 9 months, $S D=6$ months). All children were typically developing, that is, no child had any known developmental delay or neurological disorder evidenced by parental report. Two groups of young children participated, one group who was administered the TOL, and another who completed the TOH. TOL and TOH group participants were matched with respect to age, sex, and vocabulary [using either the available Picture Vocabulary subtest from the Woodcock-Johnson Cognitive Battery-Revised (Woodcock \& Johnson, 1989) or the Vocabulary subtest from the Wechsler Preschool and Primary Scales of Intelligence (Wechsler, 1990)]. A between-subjects design was used for tower task administration because involvement of executive control functions generally is considered strongest when a task is novel or difficult. Repeated encounters with tasks that are very similar in their setup and requirements (i.e., TOL and $\mathrm{TOH}$ ) would attenuate the role of executive functions in the administration of the second task (Rabbitt, 1997). Furthermore, the potential contamination between the two tasks was considered too large even with a counter-balanced presentation order. Finally, the time available for testing cognitive abilities in young children is limited; therefore, a relatively shorter battery is necessary to maximize obtaining complete data on all tasks. Tower groups did not differ in chronological age, $\mathrm{t}(116)=-.86, p$ $>.05$, sex (TOL group $=51 \%$ female, $\mathrm{TOH}$ group $=52 \%$ female; $\left.z^{2}(1, \mathrm{~N}=118)=.03, p>.05\right)$, or vocabulary, $t(116)$ $=.00, p>.05$. Independent samples $t$-tests revealed that groups were comparable on all independent or dependent measures (see Table 1), with the exception of digit span performance. Children in the $\mathrm{TOH}$ group verbally reproduced longer digit strings than children in the TOL group, $t$ (116)
$=-2.68, p<.05$. To further investigate the importance of this group difference, correlations between performance on digit span and the other EF tasks were computed for each tower group separately. Neither the strength nor the level of significance of the correlations varied substantially between the two tower groups. As the TOL and TOH groups were comparable in all other respects and task procedures were identical, the group difference in digit span was considered to be due to spurious sampling variation. Because all statistical analyses were conducted within each group and digit span performance was an independent predictor, this difference was not controlled statistically.

\section{Measures}

Tower of London (TOL; Shallice, 1982). The TOL version from the NEPSY (Korkman, Kirk, \& Kemp, 1998) was used because of its applicability for use with young children. TOL was administered in accordance with the standardized, published NEPSY instructions. Children were told they were going to play a game with three colored balls (the child was asked to name the colors of the balls). They were shown how the balls could move from one peg to another, and then told the rules for playing the game. Rules were that a) balls could only be moved one at a time; b) the balls must be kept on the pegs when they are not being moved; and c) the move is finished when their hand is taken off the ball. Children then proceeded to a practice trial (requiring the movement of only one ball). They were shown the target position in the book and asked to make their tower look like the one depicted in the book. For each experimental trial thereafter, they were told how many moves were needed to make their tower the same as the picture (between 1 and 7 moves), and that they should go as quickly as possible (time limits were 30 seconds for 1-and 2-move trials, and 45 seconds for 3-to 7-move trials; Korkman, Kirk, \& Kemp, 1998). Of the 20 trials administered, 4 involved no counter-intuitive moves, 6 required 1 counter-intuitive move, 3 required 2, 4 required 3, and 3 required 4 counter-intuitive moves. If children broke the rules, the balls were put back into their original configuration and the task resumed and the timing continued. Hence, a rule violation did not count as a failure of the item. A trial was counted as correct if children reached the target position in

Table 1. Sample demographics and performance on measures of short-term memory, inhibition, and shifting of groups administered the Tower of London versus the Tower of Hanoi

\begin{tabular}{llcc}
\hline & & $\begin{array}{c}\text { Tower of London } \\
\text { group } M(S D)\end{array}$ & $\begin{array}{c}\text { Tower of Hanoi } \\
\text { group } M(S D)\end{array}$ \\
\hline \multirow{6}{*}{ grous } & 59 & 59 \\
& Age & $4 \mathrm{y}, 8 \mathrm{~m}(4 \mathrm{~m})$ & $4 \mathrm{y}, 9 \mathrm{~m}(7 \mathrm{~m})$ \\
& Vocabulary score & $100.00(15.00)$ & $103.19(15.71)$ \\
STM & Tower score (max $=27)$ & $12.80(7.76)$ & $11.73(7.70)$ \\
Inhibition & Digit span & $3.52(0.82)$ & $4.00(1.08)$ \\
Shifting & SS Inhibit time (secs) & $27.46(15.53)$ & $28.08(15.09)$ \\
& SS Inhibit accuracy (max $=15)$ & $13.72(2.49)$ & $13.18(3.30)$ \\
& SS Shift time (secs) & $47.22(19.15)$ & $42.69(16.00)$ \\
& SS Shift accuracy (max $=15)$ & $8.10(5.34)$ & $7.64(6.63)$ \\
\hline
\end{tabular}

Note. STM = short-term memory; SS = Shape School. 
the specified number of moves and within the time limit. Testing was discontinued after 4 consecutive failures. The test-retest reliability coefficient reported in the NEPSY manual in this age group is approximately .89 (Korkman, Kirk, \& Kemp, 1998).

Tower of Hanoi (Klahr, 1978; Simon, 1975). For Tower of Hanoi (TOH), the Welsh et al. (1991) version was used. Here, the child is presented with two wooden-based models that contain 3 equal-sized, large plastic pegs. One model is described as the examiner's, where there are 3 different color rings that are stacked on the right peg. The peg diameter is graduated (Playschool ${ }^{\circledR}$ Rock-a-Stack), so that all three rings only fit on the peg stacked from largest to smallest. The child's model contains the same colored and sized rings as the examiner's, but arranged in a different configuration across the pegs. The child must move the 3 rings, one at a time, among the pegs to achieve the examiner's model configuration. Different configurations result in successively more difficult problems by increasing the number of moves that the child must make to reproduce the examiner's model configuration, the end-state goal. For preschool children, the Welsh et al. (1991) administration uses an instructional story (Klahr \& Robinson, 1981) to describe the goals and rules of the task. The story describes 'monkeys' (rings) of different sizes (Baby, Mommy, and Daddy) that may jump among the 'trees' (pegs). The goal is to 'bring the monkeys home to sleep on their tree', that is, to achieve the examiner's model. The child was told the three rules for the task, which are: a) only one monkey can move at a time; b) a bigger monkey cannot sit on a smaller monkey; c) the monkeys have to stay on the pegs if they are not in the child's hand. The child was not told the minimum number of moves required for successful completion in each trial, and the trials were not timed. Unlike Welsh et al.'s administration where 6 trials are presented for each problem, each of the 6 problems (requiring from 2 to 7 minimal moves to solve the problem) was presented just for a single trial to reduce task length in the context of the larger battery of EF tests administered. There was $1 \mathrm{TOH}$ problem with 0 counter-intuitive moves, 2 with 1 counter-intuitive move, and $3 \mathrm{TOH}$ problems with 2 counter-intuitive moves. An individual problem was discontinued upon solution or when the child made a maximum of 20 moves. Testing was discontinued after two consecutive failures, with failure occurring when the child refused to make any moves, or when they failed to make any legal moves for a given problem. Gnys and Willis (1991) reported a test-retest reliability for $\mathrm{TOH}$ of .72 in 5-year-old children; however, the test- retest interval was only 25 minutes. Bishop et al. (2001) found test-retest reliability of .53 over an interval of 30 to 40 days, in a sample of children aged 7 to 10 years.

\section{Scoring}

There are several methods to score solutions to tower problems (e.g., Krikorian, Barton, \& Gay, 1994; Klahr, 1994; Korkman, Kirk, \& Kemp, 1998; Anderson, Anderson, \& Lajoie, 1996). However, these systems capitalize on the unique TOL or $\mathrm{TOH}$ administration procedures and are not suitable to directly compare performance across the two tower tasks. In order to compare performance across the two tower tasks used here, a common dependent measure was scored from both TOL and $\mathrm{TOH}$, similar to that used by Bishop et al. (2001). Problems were assigned a point value based on how many minimal moves were required for solution (e.g., 2 move problem $=2$ points; 7 move problem $=7$ points), where the total score was the sum of all correctly solved problems up to a maximum of 7 moves. One-point problems were not used in the calculation of the total score. For example, if a child successfully solved 2-, 4-, and 5-move trials, the child achieved a total score of $11(2+4+5)$. Credit was not given for the incorrect 3-move trial. The maximal score possible for both TOL and TOH was 27 (up to 7 moves correct).

Inhibition and shifting. Two conditions (Inhibit, Shift) from the Shape School (Espy, 1997) were used to assess EF processes. This newly developed task was designed to measure and better parse executive processes in young children. In the Shape School, characters of different colors and shapes are presented to the child in a storybook format that sets up different response contingences. To establish the prepotent response bias to name the stimulus color, the child was told that the pupil's name was their color in the Control condition. Then, the child was instructed to name the pupils in order as fast as possible without making any mistakes. In the Inhibit condition, the figures showed one of two facial expressions, either happy or sad/frustrated, depending on whether the pupil was 'ready for lunch'. The child was instructed to name the pupils who were ready for lunch (i.e., happy-faced) and to not name (i.e., inhibit naming) the sad/ frustrated pupils who were not ready as quickly as possible without making any errors.

In the Shift condition, another classroom was added to the story. These pupils wore hats and their names were their figure shapes. The child was told that pupils from all classes were going to story time, and they were to name the pupils (color for pupils without hats, and shape for pupils with hats). So, this condition required flexibly shifting between response sets of color and shape. There were 15 figures in each of the Inhibit and Shift conditions. Two dependent measures were scored for each condition, total time to name all pertinent figures and accuracy (correct - errors). Preliminary normative and validity information is available (see Espy, 1997; Espy et al., 2001; Espy et al., in press), and test-retest reliability estimates are currently being established (preliminary information available from the second author upon request).

Short-term memory. Digit span was used to assess shortterm memory storage capacity. After an initial practice session, the digit sequences were presented auditorily to the child at a rate of approximately one item per 2 seconds, starting from a span length of two. Each child was required to recall the digits in the order in which they had been presented. If the items were recalled in the correct serial order, the span length was increased by one. If the child recalled the digits incorrectly, a different digit sequence of the same span length was repeated. If the child failed on the second attempt of any particular span length, testing was discontinued. The maximal digit span length was scored. 


\section{Design/Analysis}

First, correlations among the EF tasks were conducted to establish the degree of shared variance and relations to potential covariates, child vocabulary, and age. Then, a series of hierarchical regression analyses were conducted within each tower group, with Inhibition (Shape School Inhibit condition time and accuracy), Shifting (Shape School Shift condition time and accuracy), and Short-term Memory (maximal digit span) as the predictors of the respective total scores on TOL or TOH. These analyses were conducted with and without the inclusion of age, in order to examine whether the observed relations were robust across the age range studied. Finally, performance on TOL and TOH was parsed further into trials of different complexity that varied in the number of counter-intuitive moves needed to solve the problem. The number of correctly solved problems for each complexity level was calculated for each child. Then, separate regression analyses at each complexity level were conducted in the TOL and $\mathrm{TOH}$ groups, respectively, to determine the amount of variability that was related to performance on tasks of short-term memory, shifting, and inhibition.

\section{Results}

Sample performance on each tower task is shown in Table 1, clearly depicting comparable tower task proficiency across groups. To investigate the relations between tower task performance and background demographic variables, correlational analyses were conducted. Child vocabulary was unrelated to TOL, $r(57)=$ $.14, p>.05$, and TOH, $r(57)=.18, p>.05$, performance. As child vocabulary is the best predictor of general intelligence in adults and children (Sattler, 1982), this pattern is consistent with results from other developmental studies that demonstrate weak relations between $\mathrm{IQ}$ and tower and other executive function task performance (e.g., Bishop et al., 2001; Shallice, 1982; Welsh et al., 1991). However, the debate as to nature of the association between IQ and EF is still ongoing (Anderson, 2001; Duncan, Emslie, Williams, Johnson, \& Freer, 1996), particularly in adults with mature cognitive proficiencies. Because of the lack of association between child vocabulary and tower task performance, child vocabu- lary was not included in any further statistical analyses. Age, in contrast, was related to TOL performance (see Table 2), with older children solving more problems correctly. Because participation was limited to young children in a defined developmental period, the hierarchical regressions were conducted without controlling for the effect of age. When age was entered first in the regression models as a covariate, the reported results did not change substantively. The results from these analyses are available from the first author upon request.

In Table 2, a somewhat different pattern of correlations between TOL and $\mathrm{TOH}$ and digit span, Shape School inhibition scores, and Shape School shifting scores was observed. Both TOL and TOH performance was correlated positively with naming accuracy on the Shape School shift condition. Using Fisher's Z transformation, the strength of the correlations of Shape School shifting accuracy to TOL and TOH did not differ significantly (observed correlations fall within the computed confidence intervals; lower limit, $r=-.27$, upper limit, $r$ $=.44$ ). Only TOL performance was correlated negatively with latency to complete the Shape School Inhibit condition. Children who solved more TOL problems correctly took less time to name, and to inhibit naming, the pertinent stimuli in the Shape School Inhibit condition. One potential confound is that latency to complete the Shape School Inhibit condition depends in part on baseline naming speed of the stimulus colors. Partial correlation analyses were conducted, removing the latency to name the stimuli in the Shape School Control condition. In this analysis, the correlation between Shape School Inhibition latency and TOL was reduced in magnitude and was no longer significant, $r(56)=-.20, p>.05$. Neither TOL nor $\mathrm{TOH}$ performance was associated with digit span task performance. The observed pattern of relations between the EF measures and tower performance was unaltered when controlling for child vocabulary.

The inter-correlations amongst the EF measures across groups revealed that these measures do share some commonality (i.e., rather than being 'pure' measures of each EF skill), as well as diversity (e.g., Miyake et al., 2000). Children who retained and reproduced longer digit string sequences named stimuli more accurately in the Shape School Shift condition. Accuracy and time measures from the Shape School Inhibit and Shift

Table 2. Correlations among age and executive function measures with TOL and TOH

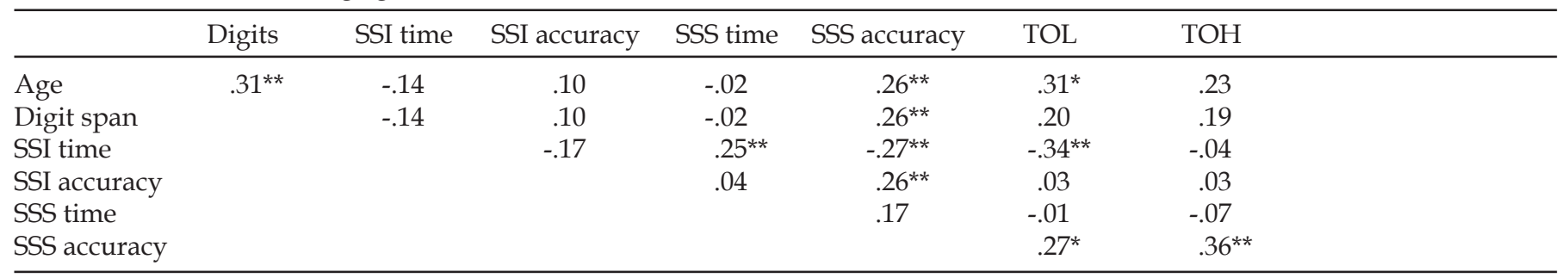

Note: SSI = Shape School Inhibit Condition, SSS = Shape School Shift Condition, TOL = Tower of London, TOH = Tower of Hanoi. For correlations with TOL and TOH, $d f=57$. For all other correlations, $d f=116 .{ }^{* *} p<.01,{ }^{*} p<.05$. 
Table 3. Results of regression analyses examining the role of executive functions in TOL and TOH performance

\begin{tabular}{|c|c|c|c|c|}
\hline & \multicolumn{2}{|c|}{ TOL } & \multicolumn{2}{|c|}{$\mathrm{TOH}$} \\
\hline & $R^{2} / R^{2} \Delta$ & F & $R^{2} / R^{2} \Delta$ & F \\
\hline 1. Inhibit & $.12^{*}$ & 3.65 & .01 & .23 \\
\hline 1. Shift & .08 & 2.48 & $.20^{* *}$ & 7.08 \\
\hline 1. STM & .05 & 2.78 & .04 & 2.29 \\
\hline \multicolumn{5}{|c|}{ Unique contribution of shifting, after controlling for inhibition and short-term memory } \\
\hline 1. Inhibit + STM & $.13^{*}$ & 2.82 & .04 & .83 \\
\hline 2. Shift & .03 & .94 & $.18^{* *}$ & 6.33 \\
\hline \multicolumn{5}{|c|}{ Unique contribution of inhibition, after controlling for shifting and short-term memory } \\
\hline 1. Shift + STM & .10 & 1.97 & $.21^{* *}$ & 4.95 \\
\hline 2. Inhibit & .07 & 2.09 & .02 & .53 \\
\hline \multicolumn{5}{|c|}{ Unique contribution of short-term memory after controlling for shifting and inhibition } \\
\hline 1. Inhibit + Shift & $.16^{*}$ & 2.50 & $.22^{* *}$ & 3.73 \\
\hline 2. STM & .01 & .42 & .01 & .78 \\
\hline
\end{tabular}

Note: $\mathrm{STM}=$ short-term memory, $\mathrm{TOL}=$ Tower of London, $\mathrm{TOH}=$ Tower of Hanoi.

${ }^{* *} p<.01,{ }^{*} p \leq .05$.

conditions also were interrelated, in addition to an association between the latency to name all stimuli in the Shape School Inhibit condition and naming accuracy in the Shape School Shift condition. This pattern of significant interrelations amongst the tasks did not change after controlling for vocabulary.

In the regression analyses, the singular contribution of Shape School inhibition scores, Shape School shifting scores, and digit span to TOL or TOH performance was examined first (i.e., as a single predictor in the regression equation - see Table 3). For TOL, Shape School inhibition was the only significant predictor of performance, accounting for $12 \%$ of the variance. In contrast, Shape School shifting was the best predictor of $\mathrm{TOH}$ performance, accounting for $20 \%$ of performance variability. Digit span was unrelated to TOL or TOH performance. Due to the confounding factor of baseline color naming speed, residualized variables were constructed for Shape School Inhibit and Shift latencies, by partialling the latency to complete the Shape School control condition. Using these residualized variables in the regression analysis eliminated the contribution of Shape School inhibition as a significant predictor of TOL performance, $R^{2} \Delta=.09$. All other results remained unchanged when the residualized variables were included in the models.

In the hierarchical regressions, the roles of Shape School inhibition and shifting and of digit span were examined as unique predictors of tower performance, that is, after statistically controlling for the effect of the other two EF measures (see Table 3). Shape School shifting scores accounted for the most unique variance in $\mathrm{TOH}$ performance, accounting for an additional $18 \%$ of the performance variability after controlling for performance on the inhibition and short-term memory tasks. For TOL, Shape School shifting did not contribute uniquely to performance, only accounting for an additional 3\% of performance variability. Shape School inhibition did not ac- count for significant, unique variance in either TOL $(7 \%)$ or TOH $(2 \%)$ performance, after accounting for performance on the Shape School shifting and digit span measures. Not surprisingly, given the singular regression model results, digit span was unrelated to either TOL or $\mathrm{TOH}$ performance, after controlling for performance on the Shape School shifting and inhibition conditions.

When the influence of trial complexity was examined, only trials involving 0,1 , or 2 counter-intuitive moves were included for comparative purposes, as very few children correctly solved problems that required more than 2 counter-intuitive moves in this age range. Table 4 contains a summary of the results of the regression analyses. Digit span accounted for small, and similar, amounts of variance across complexity levels for both TOL and TOH. For TOL, the contribution of Shape School inhibition scores to performance increased as problem complexity increased, as measured by the number of counterintuitive moves. Variance accounted for ranged from . $9 \%$, where no counter-intuitive moves were required for correct TOL solution, to $16.7 \%$, where two counter-intuitive moves were necessary. In contrast, Shape School inhibition scores accounted for a small percentage of $\mathrm{TOH}$ performance variability across problem complexity. Finally, Shape School shifting abilities accounted for comparable variance in both TOL and TOH performance. Across the problem complexity levels of 0,1 , and 2 counter-intuitive moves, shifting accounted for progressively more performance variability (TOL $=3.6 \%, 7.5 \%$, and $11.6 \%$ respectively; $\mathrm{TOH}=0 \%, 10.0 \%$, and $14.8 \%$ respectively). Therefore, whilst shifting task performance was a better predictor of performance on more complex tower trials requiring more counter-intuitive moves independent of tower task type, inhibition contributed more to complex TOL trials only. This pattern of results did not change when the residualized inhibition and shift scores were used as the predictor variables in the statistical models. 
Table 4. Contribution of short-term memory, inhibition, and shifting to prediction of TOL and $\mathrm{TOH}$ performance at increasing levels of complexity

\begin{tabular}{|c|c|c|c|c|c|c|}
\hline \multirow{2}{*}{$\begin{array}{l}\text { Complexity } \\
\text { Level }\end{array}$} & \multicolumn{3}{|c|}{ TOL } & \multicolumn{3}{|c|}{$\mathrm{TOH}$} \\
\hline & STM & Inhibition & Shifting & STM & Inhibition & Shifting \\
\hline 0 & $2.3 \%$ & $.9 \%$ & $3.6 \%$ & $.8 \%$ & $1.2 \%$ & $.0 \%$ \\
\hline 1 & $4.9 \%$ & $8.6 \%$ & $7.5 \%$ & $1.2 \%$ & $6.9 \%$ & $10.0 \%$ * \\
\hline 2 & $1.6 \%$ & $16.7 \%$ ** & $11.6 \%$ * & $5.4 \%$ & $1.2 \%$ & $14.8 \%$ * \\
\hline
\end{tabular}

Note: $\mathrm{STM}=$ short-term memory, $\mathrm{TOL}=$ Tower of London, $\mathrm{TOH}=$ Tower of Hanoi.

Complexity level refers to the number of counter-intuitive moves required for solution.

** $p<.01,{ }^{*} p<.05$.

\section{Discussion}

Overall, the results demonstrated that the ability to flexibly shift among mental sets is related to $\mathrm{TOH}$ performance. These results are consistent with the suggestions made by Bishop et al. (2001) regarding performance by school age children and adolescents, and extend the importance of mental flexibility in $\mathrm{TOH}$ performance in younger, preschool children. Furthermore, the role of shifting abilities was increasingly prominent on those trials where the child had to make more counter-intuitive moves (i.e., overcome goal-subgoal conflict) for successful completion on both tower tasks. Despite some differences in the apparatus structures that result in different task constraints, both TOL and $\mathrm{TOH}$ share certain cognitive demand characteristics, in particular the necessity to shift among subgoals that is evident on both tasks on the more complex problems.

In terms of overall performance, the role of inhibition differed between the two tower tasks. Inhibition was predictive of TOL performance when considered as a unitary predictor. Inhibition also had increasing predictive power evident on more complex TOL trials that required more counter-intuitive moves. In contrast, no significant associations were found between inhibition and $\mathrm{TOH}$ performance. These findings support those of Welsh et al. (1999), who found a similar discrepancy in the association of inhibition between TOL and TOH performance in adults. Considering differences in task administration, children were told the number of moves needed for each trial in TOL and told to solve the problems as quickly as possible; whereas in $\mathrm{TOH}$, they were not given any explicit instructions regarding the number of moves nor speeded performance. The time limit constraints imposed in TOL clearly contributed to this significant relation. Results from the correlational analyses revealed that only speed, and not accuracy, of the Shape School inhibit condition was related to TOL performance. Where baseline color naming speed was controlled, the relation between Shape School inhibition and TOL task performance was no longer significant. However, shared aspects of speeded performance do not account for this relation completely, as inhibi- tion still was related to performance on the TOL trials of higher complexity. Providing information as to the number of moves required may result in the child inhibiting the action response of immediately moving the balls and further promote the child to monitor the number of moves being made throughout the trial. Where no such constraint was given, as in $\mathrm{TOH}$, children may just keep moving the disks in real time, until the endgoal is reached. This issue could be investigated directly in future studies by altering the TOL procedure to include recording the number of excess moves made, by imposing a time limit for $\mathrm{TOH}$ trials, or by telling children the minimum number of moves required for each $\mathrm{TOH}$ trial.

The lack of association between inhibition and $\mathrm{TOH}$ performance in the young children studied here is consistent with that of Bishop et al. (2001) in school age children. Inhibition may be viewed as either suppression of automatically activated prepotent information (i.e., a relatively unconscious process), or as a more conscious inhibition of previously used task sets or strategies (as measured by shifting ability). Indeed, Bishop et al. noted that more complex inhibitory processes may play a role in tower task performance, rather than inhibition of prepotent responses as measured in their study (see also Welsh et al., 1999). In tower tasks, shifting continuously between subgoals is necessary, and as a consequence, a subgoal that was previously active must be inhibited. In the current study, shifting ability was the best predictor of performance on $\mathrm{TOH}$, perhaps subsuming such lower-order inhibitory processes.

Researchers have considered tower tasks to place a substantial load on short-term and working memory because of the necessity to store and retain elements of a sequential plan (Pennington, Bennetto, McAleer, \& Roberts, 1996). In the current study, short-term memory storage capacity was unrelated to either $\mathrm{TOH}$ or TOL performance, consistent with other findings by Welsh et al. (1999). However, most previous studies have utilized 'working memory' measures, which require concurrent processing and storage. Short-term memory measures information storage capacity, perhaps accounting for the differences across study find- 
ings. Visual-spatial working memory may contribute more strongly to tower performance in adults (e.g., Gilhooly, Wynn, Phillips, Logie, \& Della Sala, in press; Numminen, Lehto, \& Rouppila, 2001; Phillips et al., 1999), as adult participants may use a form of mental visualized movement between the pegs when planning the sequence of moves (Welsh, Cicerello, Cuneo, \& Brennan, 1995). Whilst the potential importance of visual-spatial working memory in tower performance has been reported with normal adults and adult clinical populations, the extrapolation of these findings to young children is problematic. Further research is needed to assess the contribution of visual-spatial working memory to tower task performance in young children. Based on behavioral observations during task performance, young children do not appear overtly to pre-plan their moves before implementation, as they do not pause before moving the disks/balls or do not appear to be implementing systematic move sequences. Instead, younger children may rely more on a perceptual strategy of deciding the move sequence on-line. As such, visual-spatial working memory, or indeed short-term memory, would not play such a crucial role in performance, particularly on trials where only a small number of subgoals are required (Goel et al., 2001; Shum et al., 2000).

More generally, these findings highlight the utility of this approach to better characterize the multifactorial executive functions that comprise tower task performance. There remains considerable debate as to the demand characteristics of many executive tasks. Using a shared set of predictors to directly compare performance between differing EF tasks is one method by which to elucidate the cognitive processes that contribute to performance. Unfortunately, this method cannot illuminate the particular direction of causality, as this design is correlational in nature. Longitudinal studies characterizing the dynamic relations among developing EF constructs would be more useful in this regard.

There also are limitations with this approach. Determining the underlying measurement characteristics of EF tasks is particularly complicated because the 'executive' aspect of the construct inherently involves coordinated, higher-order control of more modular processes, such as language or visual spatial abilities. EF tasks, then, will not be 'pure' measures of a specific or singular executive process. In the current study, there were correlations among the EF tasks, although the magnitude was not so high as to indicate task redundancy. Because only shifting was a unique predictor of $\mathrm{TOH}$ performance, both when entered in isolation and after accounting for the contributions of short-term memory and inhibition, its centrality in $\mathrm{TOH}$ performance was well established. In contrast, inhibition may share more overlapping variance, particularly in relation to TOL, as its contribution to TOL performance was no longer apparent when shifting and short-term memory capacity were controlled statistically. Although scientists persist in trying to isolate more cognitively simple, 'purer' tasks that specifically assess just one aspect of executive function, such efforts have proven elusive. Developing 'pure' measures for use with preschool children may be particularly problematic, as their cognitive abilities are considered less differentiated. Structural equation modeling approaches that allow for relations between latent processing constructs may yield new insights into this problem. These approaches would allow examination of differing causal models that move beyond the associations studied here. Such an approach has been used with adult samples (Miyake et al., 2000); however, the limitations on the number of measures per construct make the application of these methods more difficult in young children (Senn, Espy \& Kaufmann, in press).

In summary, shifting or mental flexibility was related more strongly to $\mathrm{TOH}$ performance in this age range, although such cognitive demands for mental flexibility on complex trials of both TOL and $\mathrm{TOH}$ were similar in young children. The role of inhibition was more prominent for TOL; however, the different instructional sets may have contributed to the observed pattern. These findings suggest that TOL and $\mathrm{TOH}$ share some communality, but are not interchangeable or isomorphic in young children. This study also raises important methodological and theoretical issues in understanding the relative contributions of differing executive functions, such as inhibition and shifting, to tower task performance. Using different dependent variables from the tower tasks, such as the number of excess moves made, latency to implement the first move, time to complete the solution, rule violations, and error types, may yield a different pattern of relations to executive skills. Because of the differing instructions and physical set-up in the two tower tasks, comparable information of this nature was not available across tasks. However, this information may provide further insight into the strategies that children use to complete tower tasks, such as on-line perceptual moves versus pre-planning, where different strategies may depend more or less on varying executive processes. Generally, even young children below 6 years of age can engage more complex executive functions, such as inhibition and shifting or mental flexibility, that impact on children's problemsolving and planning skills. In young children, these component executive abilities may be less differentiated, thereby attenuating the impact of task demand characteristics on tower task performance. Clearly, the relations among putative EF constructs and observed EF task performance need to be explicated carefully in the developmental context. Such relations will not be isomorphic in adults, adolescents, school age children, 
or preschoolers. Finally, these results do not address whether both TOL and TOH are 'prefrontal' tasks, particularly in the age range studied. Event-related potential recordings using high-density geodesic electrode nets might be a useful tool with which to investigate such questions in young children.

\section{Acknowledgments}

This study was supported by a grant to the first author from the British Academy, and by grants from the Rita Rudel Foundation; the Office of Research Development and Administration, Southern Illinois University, Special Research Program; and from the University of Arizona Foundation, to the second author. The authors thank all of the children, families, and participating schools who made this study possible. The assistance of Mary Cwik, Melanie McDiarmid, Martha Glisky, and Ruth Rodway in data collection is acknowledged. We also thank Francessa Happé and the anonymous reviewers for Journal of Child Psychology and Psychiatry for their insightful comments on the original manuscript.

Corresponding author - Rebecca Bull, School of Psychology, William Guild Building, University of Aberdeen, Aberdeen AB24 2UB, Scotland, UK; Email: r.bull@ abdn.ac.uk

\section{References}

Anderson, M. (2001). Annotation: Conceptions of intelligence. Journal of Child Psychology and Psychiatry, 42, 287-298.

Anderson, P., Anderson, V., \& Lajoic, G. (1996). The Tower of London test: Validation and standardization for paediatric populations. The Clinical Neuropsychologist, 10, 54-65.

Anderson, V. (1998). Assessing executive functions in children: Biological, psychological, and developmental considerations. Neuropsychological Rehabilitation, 8, 319-349.

Baddeley, A.D. (1996). Exploring the central executive. Quarterly Journal of Experimental Psychology, 49A, 5-28.

Baker, S.C., Rogers, R.D., Owen, A.M., Frith, C.D., Dolan, R.J., Frackowiak, R.S.J., \& Robbins, T.W. (1996). Neural systems engaged by planning: A PET study of the Tower of London task. Neuropsychologia, 34, 515-526.

Bishop, D.V.M., Aamodt-Leaper, G., Creswell, C., McGurk, R., \& Skuse, D.H. (2001). Individual differences in cognitive planning on the Tower of Hanoi task: Neuropsychological maturity or measurement error? Journal of Child Psychology and Psychiatry, 42, 551-556.
Bull, R., Johnston, R.S., \& Roy, J.A. (1999). Exploring the roles of the visual-spatial sketch pad and central executive in children's arithmetical skills: Views from cognition and developmental neuropsychology. Developmental Neuropsychology, 15, 421-442.

Bull, R., \& Scerif, G. (2001). Executive functioning as a predictor of children's mathematics ability: Inhibition, switching, and working memory. Developmental Neuropsychology, 19, 273-293.

Carlin, D., Bonerba, J., Phipps, M., Alexander, G., Shapiro, M., \& Grafman, J. (2000). Planning impairments in frontal lobe dementia and frontal lobe lesion patients. Neuropsychologia, 38, 655-665.

Chase-Carmichael, C., Ris, M.D., Weber, A.M., \& Schefft, B.K. (1999). Neurological validity of the Wisconsin Card Sorting Test with a paediatric population. The Clinical Neuropsychologist, 13, 405- 413.

Duncan, J., Emslie, H., Williams, P., Johnson, R., \& Freer, C. (1996). Intelligence and the frontal lobes: The organisation of goal-directed behaviour. Cognitive Psychology, 30, 257-303.

Espy, K.A. (1997). The Shape School: Assessing executive function in preschool children. Developmental Neuropsychology, 13, 495-499.

Espy, K.A., Kaufmann, P.M., McDiarmid, M.D., \& Glisky, M.L. (2001). New procedures to assess executive functions in preschool children. The Clinical Neuropsychologist, 15, 46-58.

Espy, K.A., McDiarmid, M.D., Cwik, M.F. Senn, T.E., Hamby, A., \& Stalets, M.M. (in press). The contribution of executive functions to emergent mathematic skills in preschool children. Developmental Neuropsychology.

Espy, K.A., Stalets, M.M., McDiarmid, M.D. Senn, T.E., Cwik, M.F., \& Hamby, A. (2003). Executive functions in preschool children born preterm: Application of cognitive euroscience paradigms. Child Neuropsychology, 8, 83-92.

Flavell, J.H., \& Wellman, H.M. (1977). Metamemory. In R.V. Kail \& J.W. Hagen (Eds.), Perspectives on the development of memory and cognition (pp. 3-33). Hillsdale, NJ: Erlbaum.

Gathercole, S.E., \& Pickering, S.J. (2000). Working memory deficits in children with low achievements in the national curriculum at 7 years of age. British Journal of Educational Psychology, 70, 177-194.

Gilhooly, K.J., Wynn, V., Phillips, L.H., Logie, R.H., \& Della Sala, S. (in press). Visuo-spatial and verbal working memory in the five-disk Tower of London task: An individual differences approach. Thinking and Reasoning.

Gnys, J.A., \& Willis, W.G. (1991). Validation of executive function tasks with young children. Developmental Neuropsychology, 7, 487-501.

Goel, V., \& Grafman, J. (1995). Are the frontal lobes implicated in planning. functions? Interpreting data from the Tower of Hanoi. Neuropsychologia, 33, 623-642. 
Goel, V., Pullara, S.D., \& Grafman, J. (2001). A computational model of frontal lobe dysfunction: Working memory and the Tower of Hanoi task. Cognitive Sciences, 25, 287-313.

Heaton, R.K., Chelune, G.J., Talley, J.L., Kay, G.G., \& Curtiss, G. (1993). Wisconsin Card Sorting Test Manual: Revised and expanded. New York: Psychological Assessment Resources.

Humes, G.E., Welsh, M.C., Retzlaff, P.D., \& Cookson, N. (1997). Towers of Hanoi and London: Reliability and validity of two executive function tasks. Assessment, 4, 249-257.

Huttenlocher, P.R., \& Dabholkar, A.S. (1997). Regional differences in synaptogenesis in human cerebral cortex. Journal of Comparative Psychology, 387, 167-178.

Kinney, H.C., Brody, B.A., Kloman, A.S., \& Gilles, F.H. (1988). Sequence of central nervous system myelination in human infancy. Journal of Neuropathology and Experimental Neurology, 47, 217-234.

Klahr, D. (1978). Goal formation, planning, and learning by preschool problem solvers or: 'My socks are in the dryer'. In R. Siegler (Ed.), Children's thinking: What develops? (pp. 181-211). Hillside, NJ: Lawrence Erlbaum.

Klahr, D. (1994). Discovering the present by predicting the future. In M. Haith, J. Benson, R. Roberts, \& B. Pennington (Eds.), The development of future-oriented processes (pp. 177220). Chicago: University of Chicago Press.

Klahr, D., \& Robinson, M. (1981). Formal assessment of problem solving and planning processes in preschool children. Cognitive Psychology, 13, 113-148.

Korkman, M., Kirk, U., \& Kemp, S. (1998). NEPSY. A developmental neuropsychological assessment. Chicago: Psychological Corporation.

Krikorian, R., Barton J., \& Gay, N. (1994). Tower of London procedure: A standard method and developmental data. Journal of Clinical and Experimental Neuropsychology, 16, 840-850.

Levin, H.S., Song, J., Ewing-Cobbs, L., Chapman, S.B., \& Mendelsohn, D. (2001). Word fluency in relation to severity of closed head injury, associated frontal brain lesions, and age at injury in children. Neuropsychologia, 39, 122-131.

Levin, H.S., Song, J., Ewing-Cobbs, L., \& Roberson, G. (2001). Porteus maze performance following traumatic brain injury in children. Neuropsychology, 15, 557-567.

Lezak, M. (1993). Neuropsychological assessment. New York: Oxford.

Lyon, G.R., \& Krasnegor, N.A. (1996). Attention, memory, and executive function. Baltimore: Paul H Brookes Publishing Company.

Miyake, A., Friedman, N.P., Emerson, M.J., Witzki, A.H., Howerter, A., \& Wager, T.D. (2000). The unity and diversity of executive functions and their contributions to complex frontal lobe tasks: A latent variable analysis. Cognitive Psychology, 41, 49-100.

Morris, R.G., Ahmed, S., Syed, G.M., \& Toone, B.K. (1993). Neural correlates of planning ability: Frontal lobe activa- tion during the Tower of London test. Neuropsychologia, 31, 1367-1378.

Numminen, H., Lehto, J.E., \& Ruoppila, I. (2001). Tower of Hanoi and working memory in adult persons with intellectual disability. Research in Developmental Disabilities, 22, 373-387.

Owen, A.M., Downes, J.J., Sahakian, B.J., Polkey, C.E., \& Robbins, T. (1990). Planning and spatial working memory following frontal lobe lesions in man. Neuropsychologia, 28, 1021-1034.

Ozonoff, S., \& Jensen, J. (1999). Brief report: Specific executive function profiles in three neurodevelopmental disorders. Journal of Autism and Developmental Disorders, 29, 171-177.

Pennington, B.F. (1997). Using genetics to dissect cognition. American Journal of Human Genetics, 60, 13-16.

Pennington, B.F., Bennetto, L., McAleer, O., \& Roberts, R.J. Jr. (1996). Executive functions and working memory: Theoretical and measurement issues. In G.R. Lyon \& N.A. Krasnegor (Eds.), Attention, memory, and executive function (pp. 327-348). Baltimore: Brookes.

Phillips, L.H., Wynn, V., Gilhooly, K.J., Della Sala, S., \& Logie, R.H. (1999). The role of memory in the Tower of London task. Memory, 7, 209-231.

Rabbitt, P. (1997). Methodology of frontal and executive function. Hove, UK: Psychology Press.

Rowe, J.B., Owen, A.M., Johnsrude, I.S., \& Passing-ham, R.E. (2001). Imaging the mental components of a planning task. Neuropsychologia, 39, 315-327.

Senn, T.E., Espy, K.A., \& Kaufmann, P.M. (in press). Using path analysis to understand executive function organization in preschool children. Developmental Neuropsychology.

Shallice, T. (1982). Specific impairments of planning. Philosophical Transactions of the Royal Society of London, B, 298, 199-209.

Shallice, T. (1990). From neuropsychology to mental structure. New York: Cambridge University Press.

Shum, D., Short, L., Tunstall, J., O'Gorman, J.G., Wallace, G., Shephard, K., \& Murray, R. (2000). Performance of children with traumatic brain injury on a 4-disk version of the Tower of London and the Porteus Maze. Brain and Cognition, 44, 59-62.

Simon, H.A. (1975). The functional equivalence of problem solving skills. Cognitive Psychology, 7, 268-288.

Stuss, D.T. (1992). Biological and psychological development of executive functions. Brain and Cognition, 20, 8-23.

Swanson, H.L., \& Ashbaker, M.H. (2000). Working memory, short-term memory, speech rate, word recognition and reading comprehension in learning disabled readers: Does the executive system have a role? Intelligence, 28, 1-30.

Swanson, H.L., Ashbaker, M.H., \& Lee, C. (1996). Learning disabled readers. working memory as a function of processing demands. Journal of Experimental Child Psychology, 61, 242-275. 
Thatcher, R.W. (1991). Maturation of the human frontal lobes. Physiological evidence for staging. Developmental Neuropsychology, 7, 397-419.

van den Heuvel, O.A., Groenewegen, H.J., Barkhof, F., Lazeron, R.H.C., van Dyck, R., \& Veltman, D.J. (2003). Frontostriatal system in planning complexity: A parametric functional magnetic resonance version of Tower of London task. Neuroimage, 18, 367-374.

Wechsler, D.H. (1990). Wechsler Preschool and Primary Scale of Intelligence-Revised. Kent: The Psychological Corporation.

Welsh, M.C., Cicerello, A., Cuneo, R., \& Brennan, M. (1995). Error and temporal patterns in Tower of Hanoi performance: Cognitive mechanisms and individual differences. Journal of General Psychology, 122, 69-81.
Welsh, M.C., Pennington, B.F., \& Groisser, D.B. (1991). A normative-developmental study of executive function: A window on prefrontal function in children. Developmental Neuropsychology, 7, 131-149.

Welsh, M.C., Satterlee-Cartmell, T., \& Stine, M. (1999). Towers of Hanoi and London: Contribution of working memory and inhibition to performance. Brain and Cognition, 41, 231-242.

Woodcock, R.W., \& Johnson, M.B. (1989). Woodcock-Johnson Cognitive Battery-Revised. Allen, TX: DLM Teaching Resources. 